\title{
22
}

\section{Algebraic Specification through Expression Transformation}

\author{
M. J. Fernández Iglesias, M. Llamas Nistal \\ Área de Ingeniería Telemática \\ Dpt. de Tecnologías de las Comunicaciones \\ Campus Universitario s/n. E-36200 Vigo, Spain \\ Tel. +3486813777 . Fax +3486812116. \\ E-mail:\{manolo,martin\}@ait.uvigo.es
}

\begin{abstract}
In this paper we present a framework to help the specifier to define, handle, and gather properties of ACT-ONE-based Abstract Data Types. This framework may also be used to add data support to other formal design tasks like normalization, or verification of relations like bisimulation. This framework has been implemented in the LOTOS design tool LOLA to fulfill these objectives.
\end{abstract}

\section{Keywords}

FDT-based system and protocol engineering. Tools and tool support. Algebraic Specification. LOTOS.

\section{INTRODUCTION}

Formal Description Techniques (FDTs) provide a model for system specification whose structure usually resembles that of programming languages. A part of the language is devoted to the specification of the system's behaviour or evolution, and another part is used to specify the corresponding data structures.

These two parts are complementary. Specification styles have been defined and analyzed which put more emphasis in either behaviour or data definitions(Logrippo et al. 1992)(Vissers 1990). It can be said that behaviour specification affects the description of data structures and vice versa.

Several of the most used FDTs support a data model based on Abstract Data Types (ADTs) (e.g. LOTOS(ISO 1988)(ISO 1997) and SDL(ITU 1993)). With respect to formal specification, ADTs have several advantages: they are fully implementation independent, have enough expressive power to support formal analysis of system properties, and have a sound underlying mathematical model.

On the other side, practice has shown that ADTs have some drawbacks, mainly for prototyping or testing (i.e. as we come close to the final implementation) (Turner 1993). Although ADT's descriptive power is very suitable for the specification of ab- 
stract (implementation independent) properties of a given system, it is very difficult to map ADT descriptions to real data structures handled by real systems. Indeed, the average system designer is not required to be a mathematician trained in Universal Algebra, and consequently lacks the needed background to fully understand the consequences of an ill-constructed Algebraic Specification. This problem is not completely solved with standard libraries, because the specifier may freely extend standard data types in an inconsistent way.

In this paper we present a framework to help the specifier to define, handle, and gather properties of ADTs. This framework may also be used to add data support to other formal design tasks like normalization, or verification of relations like bisimulation. This framework has been implemented in the LOTOS design tool LOLA (Quemada 1987) to fulfill the objectives described here.

\section{TOOL DESCRIPTION}

Usually, LOTOS ADTs are interpreted as rewrite systems(Mitchell 1996) and rewriting is used as the only tool to process data expressions. This significatively reduces the application field, and does not permit abstract analysis of general data properties. For example, we can only assess if a data expression is valid for concrete instantiations of its free variables, but we cannot perform other tasks like obtaining a solution set, or conditioning data expressions for behaviour analysis.

Sometimes (e.g. SMILE(Eertink et al. 1992)), a narrowing tool is used to extend the functionality offered by a rewriting tool. Typically, a narrowing tool takes an equational theorem as input and tries to produce a solution set for it. This helps to reduce the state space during symbolic execution detecting unsolvable boolean predicates, and assists the environment (i.e. the specifier) to assign values to variables.

In both cases, standard tools from equational reasoning are used basically to assess if a boolean predicate is valid in a given theory. On the other side, we propose a framework specifically designed to assist the specifier along the whole design process. We tried to design a tool which permits the specifier to study the properties of the ADTs being defined. For example, to see the (side) effects of introducing new equations, to analyze boolean predicates which affect the evolution of the system, etc.

Furthermore, this framework has been constructed to support automatic transformation of data expressions as a subtask of behaviour transformation. Behaviour transformation may be related to design tasks like normalization, test generation or verification. As a consequence, the data transformation process should be easily configured to obtain the most suitable data expressions in a given context.

The whole process is based on the transformation of an initial boolean predicate (equational theorems are particular cases) driven by a set of transformation rules. On each transformation step, a target expression is transformed into an equivalent one applying the rules defined below.

In the next paragraphs we describe this set of rules and the corresponding control strategy. Then, we provide some examples on the use of the proposed technique in 
contexts related to the FDT-based design process. At the end of this paper, we present some conclusions.

\subsection{Basic Definitions}

The boolean operators true, false, and, and or are represented respectively as $T$, $\perp,(\cdot, \Pi)$ and $\left(+, \sum\right)$.

A signature is a pair $\Sigma=(S, \Omega)$, where $S$ is a set of sorts and $\Omega$ is a set of $S^{+}$sorted operation symbols. $X$ is an $S$-sorted set of variables. $\operatorname{Var}(t) \subset X$ is the set of variables in term $t$. A term $t$ with $\operatorname{Var}(t)=\emptyset$ is called ground. The symbol " $\emptyset$ " denotes an empty set. $t$ is said to be linear if each variable in $t$ appears only once.

For each sort $s \in S$ an (infix) boolean-sorted operation $=_{s}$ which represent semantic equivalence in $s$ is assumed. $b$ represents the sort for boolean operation symbols.

Contexts and positions are denoted as usual. $t \mid p$ represent the sub-term of $t$ at position $p . t[p \leftarrow s]$ represents a term $t$ whose sub-term at position $p$ has been replaced by term $s$.

Substitutions are endomorphisms that extend mappings from variables to the set of terms. (Variable) renamings are mappings from variables to variables. If $\rho$ is a bijective renaming and $t$ a term, the term $\rho t$ is said to be a variant of $t$. We will also use an alternative representation for substitutions, based on the solved forms introduced in (Martelli et al. 1982). That is, the substitution $\sigma=\left\{t_{1} \rightarrow x_{1}, \ldots t_{n} \rightarrow x_{n}\right\}$ is represented as the boolean predicate $\prod_{i=1}^{n}\left(x_{i}={ }_{s_{i}} t_{i}\right)$.

Substitution $\iota$ represents the identity substitution: $\iota t=t$.

A conditional rewrite system is a pair $\mathcal{R}=(\Sigma, R)$, where $\Sigma$ is a signature and $R$ a set of conditional rewrite rules $R=\left\{\prod_{j=1}^{n} P_{j i}: l_{i} \rightarrow r_{i}\right\}$. The boolean terms $P_{j i}$ are called premises. $l_{i}$ is a search pattern and $r_{i}$ a substitution pattern. A rewrite rule is said to be left linear if the corresponding search pattern is linear. A rewrite system $\mathcal{R}$ is said to be left linear if all rewrite rules in $R$ are left linear. Termination, confluence and convergence are defined as usual(Mitchell 1996). We write $s \stackrel{r !}{\longrightarrow} t$ if $s$ rewrites to $t$ and $t$ is a normal form.

The set $\Omega_{c} \subseteq \Omega$ is the set of constructors. Constructors are operation symbols that do not appear in any outermost position of a search pattern.

Definition 1 Let $C_{l}=\left\{c_{i}: l \rightarrow r_{i} \in R \mid \rho_{i} l_{i}=l\right\}$ where the $\rho_{i}$ are bijective renamings. $C_{l}$ is said to be completely defined if and only if for all substitutions $\sigma$ such that $\operatorname{Var}\left(\sigma c_{i}\right)=\varnothing$ we have that $\sum_{i}^{\operatorname{Card}\left(C_{l}\right)} \sigma c_{i} \stackrel{r !}{\longrightarrow} \mathrm{T}$, and $\sigma c_{i} \cdot \sigma c_{j} \stackrel{r !}{\longrightarrow} \perp$ for all $i \neq j$.

$R=\bigcup_{i} C_{l}^{i}$ is said to be completely defined if and only if every $C_{l}^{i}$ is completely defined.

$C_{l}$ is the set of all (variants of) rewrite rules which share the same search pat- 
tern $l$. Note that for a rewrite rule without premises there is always a (one element) completely defined set $C_{l}=\{l \rightarrow r\}$.

The following relation will be used to relax the requisites for variable binding during mutation (see below). Linearity for $t$ is required to obtain well-formed substitutions.

Definition 2 Let $t$ be a linear term. We define the conditioning substitution for two terms $t$ and $s\left(\sigma_{c}(t, s)\right)$ inductively as follows:

- $\sigma_{c}(t, s)=\iota$ if $t=o p_{1}\left(t_{1}, \ldots, t_{n}\right)$ and $s=o p_{2}\left(s_{1}, \ldots s_{n}\right)$, with $o p_{1} \neq o p_{2}$

- $\sigma_{c}(t, s)=\iota$ if $s, t$ are both ground.

- $\sigma_{c}(t, s)=\iota$ if $t \notin X$ and $s \in X$

- $\sigma_{c}(t, s)=\{s \rightarrow x\}$ if $t=x \in X$

- $\sigma_{c}(t, s)=\bigcup_{i} \sigma_{c}\left(t_{i}, s_{i}\right)$, if $t=o p_{1}\left(t_{1}, \ldots, t_{n}\right)$ and $s=o p_{2}\left(s_{1}, \ldots s_{n}\right)$, with $o p_{1}=o p_{2}$.

Example 1 Let $x_{i}, w_{i}$ be variables, and $f, g, h, m, n, a, b$, coperation symbols. Then $\sigma_{c}(t, s)=\left\{c \rightarrow x_{1}, m\left(w_{3}\right) \rightarrow x_{3}\right\}$ for

$$
\begin{aligned}
& t=f\left(g\left(h\left(x_{1}, a\right), b\right), \quad n\left(x_{2}\right), \quad x_{3}\right) \\
& s=f\left(g\left(h\left(c, w_{1}\right), b\right), \quad w_{2}, \quad m\left(w_{3}\right)\right)
\end{aligned}
$$

\subsection{Expression Representation}

To be transformed expressions are represented as sums of boolean products (SBP). An SBP is a boolean expression of the form $\sum_{i} S_{i}$, where each $S_{i}$ is a boolean product $S_{i}=\prod_{j} P_{i j}$. The $P_{i j}$ are boolean predicates which do not contain the operators + (boolean or) and - (boolean and). The $P_{i j}$ are named target predicates.

Every boolean expression can be straightforwardly rewritten into an SBP using the usual rewrite rules for boolean operators. Equations are boolean predicates of the form $\left(t_{1}={ }_{s} t_{2}\right)$

Our objective is to transform an SBP $e$ into an equivalent SBP $e_{t}$. When the proposed technique is applied to equation solving, solution construction can be easily supervised. In a given stage of the process, a set of solutions can be represented as an SBP (one product representing each solution). We can also apply the properties of boolean algebra to rearrange or transform the solution set into an equivalent one satisfying some required properties for a given application context. Furthermore, the transformation process can be halted at any point with an expression equivalent to 
the initial one. This permits also to easily represent incomplete and parameterized solutions.

To sum up, boolean expressions will be eventually transformed into an equivalent

- ground term. If this term isT, we conclude that the starting SBP is satisfied for every variable assignment. For an initial expression $\left(t_{1}={ }_{s} t_{2}\right)$, the result is the proof of the corresponding equational theorem. If this term is $\perp$, the initial SBP is never satisfied.

- new SBP. For an initial equational theorem, each product in the transformed expression represents a solution to the starting equation (i.e. conditions that must be satisfied for the theorem to be valid). Unification(Hussman 1985)) can be seen as a particular case of expression transformation. If $\mathcal{R}$ is adequately constructed, a goal $\left(t_{1}={ }_{s} t_{2}\right)$ can be transformed into an expression $\sum_{i=1}^{m} \prod_{j=1}^{n}\left(x_{j}=u_{i j}\right)$, where the $x_{j}$ are the variables of the goal, and the substitutions $\sigma_{i}=\prod_{j=1}^{n}\left(x_{j}=\right.$ $u_{i j}$ ) represent the corresponding unifiers.

\subsection{Rule Description}

The process is driven by a set of transformation rules. Associativity and commutativity of variable assignments and boolean operators $\Pi$ and $\sum$ is assumed. Rules are applied to target predicates in $S$. The transformation process is based on a convergent, completely defined and left linear rewrite system $\mathcal{R}$.

The rules are presented in Table 1 . Rule 1 (rewriting) is used to provide normalized expressions to the rest of the rules. Consequently, we will assume that the rest of the rules transform normalized expressions.

Rules 2, 3 and 4 are used to eliminate the top-level operation symbol. Let $t$ be a non-ground, normalized term. If the top-level operation symbol is not a constructor, rule 2 is applied. If this symbol is a constructor, we apply rules 3 or 4 depending on the shape of the target.

Rule 2 (mutation) is based on the properties of narrowing and boolean algebra. An equivalent expression is obtained by mutation. Further processing is simplified due to the properties of the conditioning substitution $\sigma_{c} . R_{o p}$ is supposed to be constructed from fresh variants of rewrite rules. As a particular case, if the expression to be transformed is a variable assignment $\left(x={ }_{s} t\right)$, the target $\left(t==_{s} x\right)$ is transformed instead.

In a typical narrowing process, backtracking is used to recover a previous state when a sequence of rewrite rule applications leads to no solution. In our case, all available information which leads to a solution is introduced in the target expression. This allows us to halt the transformation process at any point with an equivalent expression, and consequently to support an interactive tool for expression transformation.

Rule 3 (decomposition) is based on the properties of constructors. If the outermost operation symbols in $t_{1}$ and $t_{2}$ are both constructors, the corresponding target can 
Table 1 Transformation rules

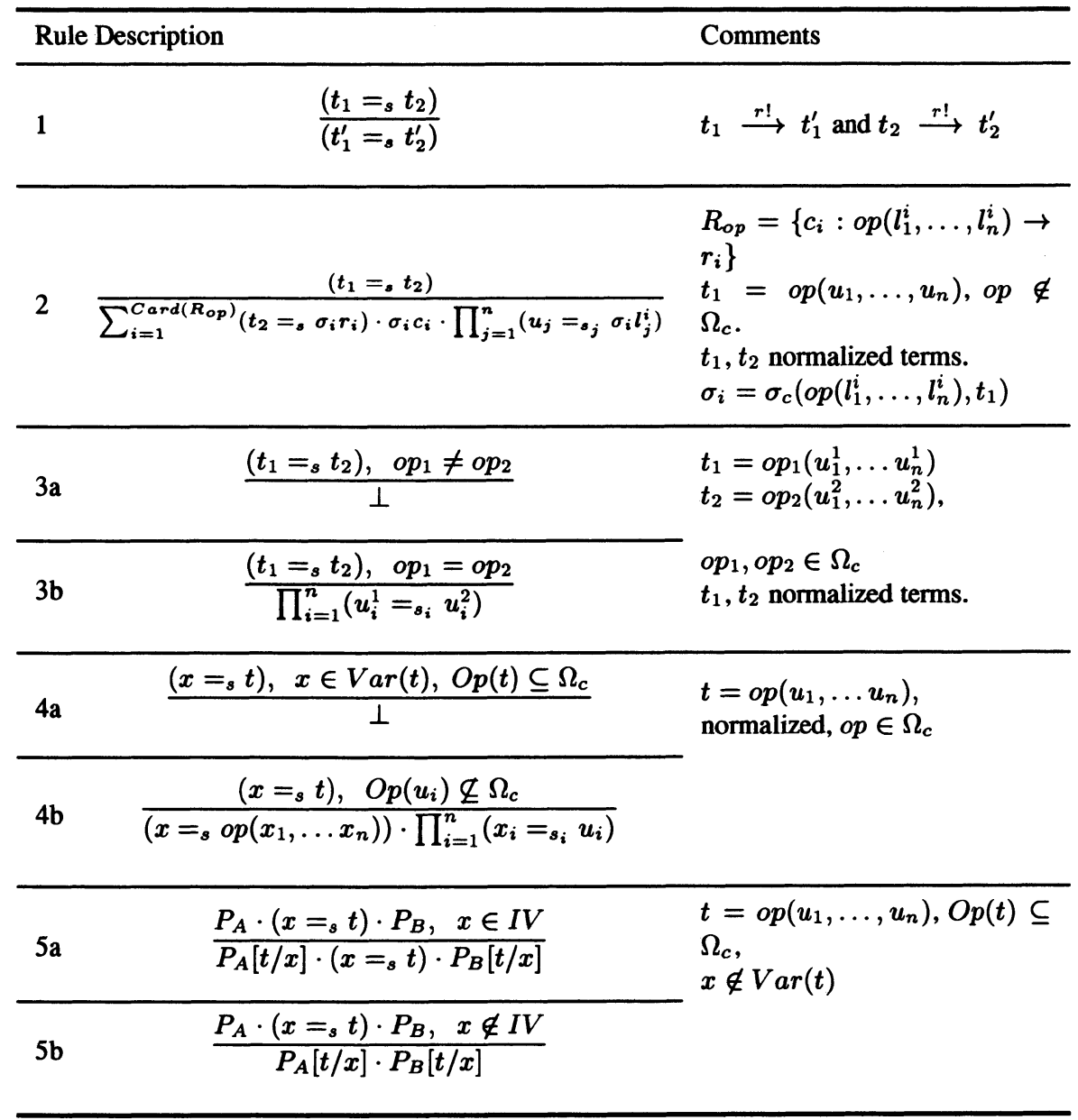

lead to a solution only if both constructors are the same. In this case, subterms must be unified one to one.

Rule 4 (imitation) is used to transform a variable assignment with an outermost constructor symbol. Rule $4 \mathrm{a}$ is correct because only finite terms and well-formed constructors are considered. Using rule $4 \mathrm{~b}$ we obtain a substitution which permits to rebuild the initial target, together with a new variable assignment which is normalized with respect to rewriting. The subterms of the obtained substitution may be further transformed. $O p(t)$ is the set of operation symbols in term $t$.

Rule 5 (variable simplification) is used to discard duplicate variable assignments 
and to detect inconsistencies (e.g $\left(x=_{s} a\right)\left(x={ }_{s} b\right)$ provided that $\left.\left(a \neq_{s} b\right)\right) . I V$ is the set if variables belonging to the initial SBP.

Example 2 The following rewrite rules provide the classical definition of the list append operation. List elements belong to sort $e, l$ is the list sort. cons is the list constructor. nil represents an empty list.

$$
\begin{aligned}
& \operatorname{app}(\operatorname{nil}, x) \rightarrow x \\
& \operatorname{app}(\operatorname{cons}(a, x), y) \rightarrow \operatorname{cons}(a, \operatorname{app}(x, y))
\end{aligned}
$$

Our goal is to transform a target $\left(\operatorname{app}\left(x_{1}, x_{2}\right)==_{l}\right.$ cons $\left(e_{1}\right.$, cons $\left.\left.\left(e_{0}, n i l\right)\right)\right)$ using the proposed rules. The transformation process evolves as presented in Figure 1 (expressions being transformed in the next step are boxed, stable expressions (marked as $\dagger)$ are not repeated in further transformation steps, $a_{i}, z_{i}$ are variables).

$$
\begin{aligned}
& \text { Rule Expression } \\
& \text { - } \quad\left(\operatorname{app}\left(x_{1}, x_{2}\right)={ }_{l} \operatorname{cons}\left(e_{1}, \operatorname{cons}\left(e_{0}, n i l\right)\right)\right) \\
& 2 \quad\left(x_{2}=l \text { cons }\left(e_{1}, \text { cons }\left(e_{0}, n i l\right)\right)\right)\left(x_{1}=l n i l\right)^{\dagger}+ \\
& 3 \quad\left(\operatorname{cons}\left(a_{1}, \operatorname{app}\left(z_{1}, x_{2}\right)\right)==_{l} \operatorname{cons}\left(e_{1}, \operatorname{cons}\left(e_{0}, n i l\right)\right)\right)\left(x_{1}=_{l} \operatorname{cons}\left(a_{1}, z_{1}\right)\right) \\
& 5 \quad\left(\operatorname{app}\left(z_{1}, x_{2}\right)==_{l} \operatorname{cons}\left(e_{0}, n i l\right)\right)\left(x_{1}=l \operatorname{cons}\left(e_{1}, z_{1}\right)\right) \\
& 2 \quad\left(z_{1}={ }_{l} n i l\right)\left(x_{2}={ }_{l} \operatorname{cons}\left(e_{0}, n i l\right)\right)\left(x_{1}={ }_{l} \operatorname{cons}\left(e_{1}, z_{1}\right)\right)+ \\
& \text { 2, 3, } 5 \quad\left(x_{1}=l \text { cons }\left(e_{1}, \text { cons }\left(e_{0}, n i l\right)\right)\right)\left(x_{2}=l n i l\right)^{\dagger}
\end{aligned}
$$

Figure 1 Transformation process for $\left(\operatorname{app}\left(x_{1}, x_{2}\right)={ }_{l} \operatorname{cons}\left(e_{1}, \operatorname{cons}\left(e_{0}, n i l\right)\right)\right)$

The final expression is

$\left(\left(x_{2}==_{l} \operatorname{cons}\left(e_{1}, \operatorname{cons}\left(e_{0}, n i l\right)\right)\right) \cdot\left(x_{1}={ }_{l} n i l\right)\right)+$

$\left(\left(x_{2}=l \operatorname{cons}\left(e_{o}, n i l\right)\right) \cdot\left(x_{1}={ }_{l} \operatorname{cons}\left(e_{1}, n i l\right)\right)\right)+$

$\left(\left(x_{1}={ }_{l} \operatorname{cons}\left(e_{1}, \operatorname{cons}\left(e_{0}, n i l\right)\right)\right) \cdot\left(x_{2}={ }_{l} n i l\right)\right)$

This expression, equivalent to the initial one, can be seen as the solution set of the initial expression $\left(\operatorname{app}\left(x_{1}, x_{2}\right)={ }_{l} \operatorname{cons}\left(e_{1}, \operatorname{cons}\left(e_{0}, n i l\right)\right)\right)$

\subsection{Control Strategy}

The transformation process is performed through successive transformation steps. Each step starts with an SBP and terminates with an equivalent SBP.

For each step, transformation rules are selected and applied to one target predicate from the active SBP. Target predicates are marked as stable (no more transformation steps will be performed for the corresponding target), unstable (the target may be 
further transformed) or loop (the target stems from a syntactically equivalent target through previous transformation steps).

Each transformation step is performed as follows:

1. The active SBP is simplified using usual rewrite rules for booleans (Fernández 1997). Commutativity and associativity of boolean operators is applied implicitly. During this process, rule 1 (rewriting) is applied to all unstable SBPs. Rewritten ground terms are marked as stable.

2. The first unstable SBP is selected, and one of rules 2 (mutation), 3 (decomposition) and 4 (imitation) is applied depending on the shape of the target as described above. Loops in targets are detected and marked accordingly (see below).

Rule 2 may generate new products. These products are appended to the right of the active SBP and the new targets are marked as unstable.

3. Rule 5 (variable simplification) is applied to the whole product where the target was taken.

Target predicates are selected left to right from the active SBP, and the process continues until no more targets are available, or a step limit, configured by the user, is reached. As we have an expression equivalent to the initial predicate at the beginning of every transformation step, we may adapt the target selection process to fulfill the requirements of an underlying behaviour analysis (variable separation, normalization, factorization, etc.). We may also halt the transformation process at the beginning of every transformation step to ask the specifier for the next target. This permits the implementation of an interactive transformation tool where the designer may make use of a prior knowledge of the problem to configure the best transformation strategy for a given design task.

With the transformation rules and the control above, infinite derivation sequences may be generated during mutation (application of rule 2). After the application of these rules, we check if any of the new predicates is a variant of a previously mutated ancestor. If this happens, the new expression is marked as loop and is not further transformed.

\subsection{Correctness}

The proposed set of rules in Table 1 together with the simplification process described in point 1 . of section 2.4 above is sound and complete for rewrite systems which have the properties enumerated in section 2.3 (Fernández 1997). Nevertheless, theoretical correctness does not guarantee termination.

Furthermore, this framework should be applicable in environments where these properties are not fulfilled. For instance, it would be desirable that a specifier who is defining abstract data types can see the behaviour of the produced data definitions to correct or improve them. The final objective is to provide a tool for the specifier to 
assess if data descriptions indeed define the wanted functionality. This is the reason to introduce the above outlined control strategy and loop detection.

\section{EXAMPLES}

The proposed examples are based on the LOTOS(ISO 1988) ADT description appearing in Figure 2. This LOTOS ADT specifies data for the transfer of primitives between a set of client nodes and a central hub. Natural numbers and booleans are specified as usual (c.f. standard library in (ISO 1988)). Although this description is fairly simple, we think that it is adequate enough for our purpose, that is, illustrate how a transformation-oriented data tool may be useful for an FDT-based design process.

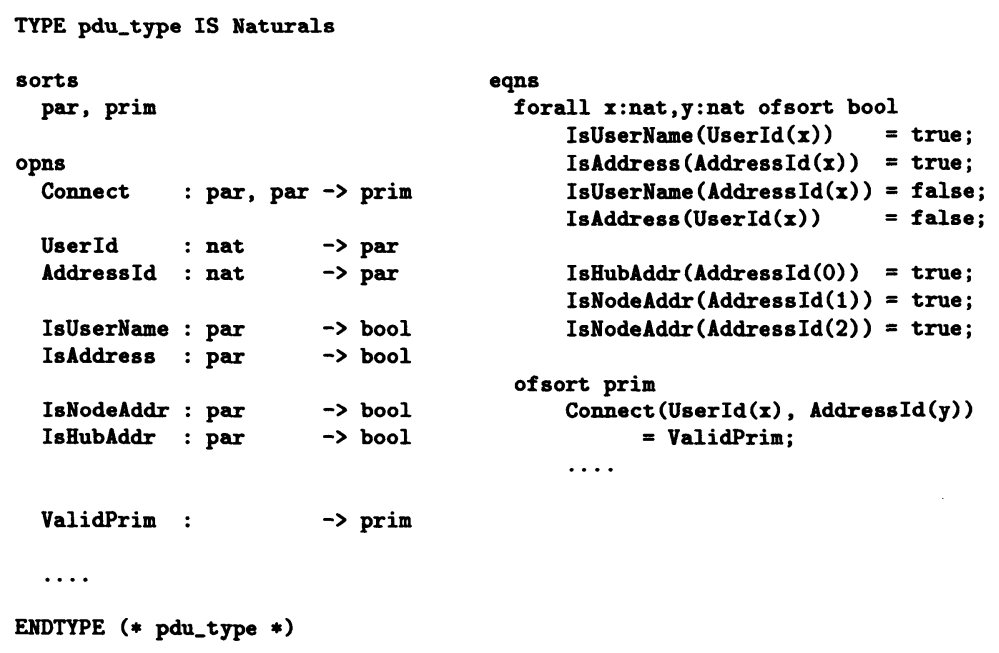

Figure 2 ADT description for the examples.

Example 3 We have the following LOTOS behaviour expression:

a?x: nat; (b!IsNodeAddr (AddressId (x)); NodeConn (x) []

b! IsHubAddr (AddressId (x)); HubConn (x))

The systems takes from the environment the information needed to construct an address id. Further system evolution depends on the kind of address constructed (i.e., a node address or a hub address).

Let us suppose that along the design process a new requirement is introduced which states that the address space of the hub is included in the address space of 
the rest of the nodes. That is, the equation IsNodeAddr (AddressId(0)) = true is appended to the equation set.

In this case, the data transformation tool transforms the predicate

$$
\text { Is NodeAddr }(\operatorname{AddressId}(x))={ }_{\text {bool }} I s H u b A d d r(\operatorname{AddressId}(x))
$$

into the equivalent predicate $\left(x={ }_{n a t} 0\right)$. The specifier sees that if $\left(x={ }_{n a t} 0\right)$ the evolution of the system is not determined because the environment may synchronize in both branches. The specifier may use this information to analyze the introduced requirements and test if they describe the desired functionality.

In the example above we can see that it is not needed to instantiate $x$ nor a (dynamic) testing procedure to detect the side effects of the new requirement. This analysis is performed statically. Data definitions affect system evolution and, as a consequence, abstract reasoning on data definitions may be useful even at the early stages of the design process.

Example 4 If we transform the predicate (Connect $(x, y)={ }_{\text {prim }}$ ValidPrim) we obtain the equivalent expression $\left(x=_{\text {par }} U \operatorname{ser} I d\left(x^{\prime}\right)\right) \cdot\left(y==_{\text {par }} \operatorname{AddressId}\left(y^{\prime}\right)\right)$, This predicate specify conditions that must be fulfilled in order to satisfy the initial expression.

In other words, for Connect $(x, y)$ being a valid primitive, the corresponding parameters must be respectively a well-constructed user id. and a well-constructed address id.

The specifier may use the proposed tool to see if the defined data indeed specify the desired functionality.

Example 5 A specifier sees unexpected results during the transformation of LOTOS specifications based on the ADT definitions in Figure 2. Specifically, observes that in some interactions with the environment, the parameters of connection primitives are lost.

She/He decides to use an interactive data transformation tool to analyze the properties of Connect. To start with, she/he tries to analyze when two connection primitives are seen as equivalent. The following initial predicate is transformed interactively:

$$
\operatorname{Connect}(x, y)={ }_{\text {prim }} \operatorname{Connect}(w, z)
$$

After a transformation step, (mutation of Connect $(x, y)$ applying the rewrite rule for Connect in Figure 2 using the substitution $\sigma=\left\{x \leftarrow U \operatorname{serId}\left(x^{\prime}\right), y \leftarrow\right.$ AddressId $\left.\left(y^{\prime}\right)\right\}$ ) obtains:

$\left(x={ }_{p a r} U \operatorname{ser} I d\left(x^{\prime}\right)\right) \cdot\left(y={ }_{\text {par }} \operatorname{AddressId}\left(y^{\prime}\right)\right) \cdot\left(\operatorname{Connect}(w, z)=_{\text {prim }}\right.$ ValidPrim $)$ 
The specifier selects the predicate $\left(\right.$ Connect $(w, z)={ }_{\text {prim }}$ ValidPrim) for transformation to obtain

$$
\begin{aligned}
& \left(x={ }_{\text {par }} U \operatorname{serId}\left(x^{\prime}\right)\right) \cdot\left(y==_{\text {par }} \text { AddressId }\left(y^{\prime}\right)\right) \cdot \\
& \left(w=\text { par } U \operatorname{serId}\left(w^{\prime}\right)\right) \cdot\left(z==_{\text {par }} \text { AddressId }\left(z^{\prime}\right)\right) \cdot \\
& \left(\text { ValidPrim }=_{\text {prim }} \text { ValidPrim }\right)
\end{aligned}
$$

The specifier detects that all connection primitives are equivalent if the corresponding parameters are well-constructed. Next, the specifier eliminates the rule which produced the mutation and transforms the initial expression again. In this case, the predicate $\left(x={ }_{p a r} w\right) \cdot\left(y={ }_{p a r} z\right)$ is obtained.

That is, two connection primitives are equivalent if and only if the corresponding parameters are also equivalent. The designer may go on with this process to configure the most suitable data model for a given problem.

In this example we can see that the proposed framework is robust enough to handle incomplete, even inconsistent, data definitions. The specifier can analyze intuitively the consequences of the defined semantics.

\section{CONCLUSIONS}

In this paper we have proposed a framework to help the designer to define, handle and gather properties of Abstract Data Type specifications. As the system behaviour depends on the data definitions, the proposed tool may also be used to assist the specifier in other design tasks targeted to the system as a whole.

This technique is based on a set of transformation rules and may be applied to other contexts where data expression transformation is needed (e.g. theorem proving or functional programming). In our case, this framework has been implemented in LOLA for the analysis and transformation of LOTOS data expressions.

All the information related to the transformation process is present in the same expression being transformed. Consequently, this process can be configured to obtain the most suitable data expressions for a given problem. This can be done choosing an adequate strategy to select targets from the active expression.

The transformation process can be halted at any point returning an expression equivalent to the initial one. The resulting expression (a boolean predicate) may be seen as a set of requisites that must be satisfied by the initial expression to be valid in a given theory. This process makes use of all information available in an ADT specification (even an incomplete one) to offer to the specifier different views of the semantics being defined.

\section{ACKNOWLEDGMENTS}

This work has been partially funded by the regional government of Galicia, Spain, under contract number XUGA32204B95. 


\section{REFERENCES}

Eertink, H. and Wolz, D. (1992) Symbolic Execution of LOTOS Specifications. in Formal Description Techniques V: FORTE'92 (eds. M. Diaz and R. Groz), North-Holland.

Fernández, M. J. (1997). Contribución al Tratamiento de Datos en LOTOS, Tesis Doctoral (PhD thesis), Dep. de Tecnologías de las Comunicaciones, Universidade de Vigo, Spain. (In spanish)

Hussman, H. (1985) Unification in conditional-equational theories. Lecture Notes in Computer Science, 204, Springer-Verlag.

ISO (1988) LOTOS: a Formal Description Technique based on the Temporal Ordering of Observational Behaviour, ISO International Standard 8807, TC97/SC21.

ISO (1997) Revised working draft on enhancements to LOTOS. Technical report, ISO/IEC JTC1/SC21/WG7.

ITU-T (1993) SDL: Specification and description language, CCITT Recommendation Z.100.

Logrippo, L., Faci, M. and Haj-Hussein, M. (1992) An introduction to LOTOS: Learning by examples, in Computer Networks and ISDN Systems, 23, 325342.

Martelli, A. and Montanari, U. (1982) An efficient unification algorithm. in ACM Transactions on Programming Languages and Systems, 4(2), ACM.

Quemada, J., Fernández, A. and Mañas, J.A. (1987) LOLA: Design and Verification of Protocols using LOTOS, in Ibercom, Conference on Data Communications, Lisbon, Portugal.

Mitchell, J.C. (1996) Foundations for Programming Languages, Foundations of Computing. The MIT Press.

Turner, K. J. (1993) Using formal description techniques - An introduction to Estelle LOTOS and SDL. Wiley, New York.

Vissers, C., Scollo, L., van Sinderen, W. and Brinksma, E. (1990) On the use of specification styles in the design of distributed systems, technical report, University of Twente. Faculty of Informatics, Entschede, The Netherlands.

\section{BIOGRAPHY}

Manuel J. Fernández graduated from the Universidade de Santiago de Compostela, Spain with a Ingeniero de Telecomunicación degree in 1990, and from Universidade de Vigo, Spain with a Doctor in Telecommunications degree in 1.997.

Martín Llamas received the Ingeniero de Telecomunicacion (1986) and Doctor in Telecommunications (1994) degrees from the Universidad Politécnica de Madrid, Spain.

Both joined the Telecommunication Engineering faculty of the Universidade de Vigo, Spain. In addition to teaching, they are involved in research in the areas of Formal Description Techniques and Computer Based Training. 